\title{
Health promotion: Views of practice principals
}

\author{
General health promotion in general dental practice - The involvement of the dental team \\ Part 2: A qualitative and quantitative investigation of the views of practice principals in South Yorkshire \\ T. A. Dyer and P. G. Robinson BrDent J 2006; 200: 45-51
}

Aim To investigate the factors that might influence the provision of general health promotion through seven different health interventions by dental teams in general dental practice.

Method A mixed-method was used comprising cross-sectional qualitative research using semi-structured interviews of a purposive sample of 10 practice principals, and a cross sectional survey of a practice principal from every dental practice in South Yorkshire, using a self-complete questionnaire.

Results Two core categories emerged from the qualitative data: seeing health or disease and practitioners' views of the structure of dental practice. The former refers to the participants' general outlook and cut across many dimensions constituting the structure of dental practice. Health-orientated dentists were more likely to be involved in prevention and were more open-minded to expanding the dental team's role into general health promotion. However participants perceived that barriers existed to involvement such as time and financial factors, current workload and lack of personal skills. The response rate of useable questionnaires in the cross sectional survey was $84 \%$. Reported levels of involvement in general health promotion were low. Most frequently reported barriers were 'insufficient funding' and 'poor use of time.' 'Poor use of time' and 'lack of training/knowledge' were reported less frequently for PCDs than dentists $(p<0.05)$. Most dentists agreed that PCDs could be trained to deliver health interventions and would be happy for PCDs to do so in their practice if reported barriers were removed.

Conclusions Although dental teams' involvement in general health promotion is low, there is willingness to increase involvement particularly among health-orientated dentists. Some reported barriers to involvement might be removed by impending changes to the GDS in England. Other important factors include a lack of education and workforce shortages of dentists and PCDs. Respondents indicated a high regard for PCDs and there was broad agreement that they were suitable to be involved in this work.

\section{IN BRIEF}

- Dental teams could contribute to meeting Government targets for reducing coronary heart disease and cancer.

- Dentists' views on involvement in general health promotion were influenced by whether they saw the purpose of dentistry as achieving health or treating disease. Those with more of a health focus were already involved and wanted to do more.

- Dental teams' involvement in general health promotion is lower than might be expected given reported views on its relevance to dentistry.

- Although barriers to involvement included time and financial factors, current workload and lack of personal skills, fewer dentists felt that these were barriers for PCDs.

\section{COMMENT}

In a previous paper Dyer and Robinson reviewed the evidence base for a range of brief preventive interventions delivered in primary care settings. They highlighted the potential role dental practitioners and their teams could play in supporting behaviour change amongst their patients. Published evidence is of course an important element in developing a preventive approach in general dental practice. However it is also essential that the views and experiences of practitioners are assessed to determine how such an approach could be implemented effectively.

Using a mixed methodology, the aim of this study was to investigate the factors that might influence the provision of health advice in seven areas of preventive care by dental teams across South Yorkshire. A range of interesting and encouraging findings are reported which largely support the existing literature. Any efforts to encourage dental practitioners to become more actively engaged in prevention need to acknowledge the range and diversity of factors influencing clinical behaviour. If we wish to make the preventive choices, the easier choices for dental practitioners and their teams, the barriers blocking this reorientation need to be addressed. For example, some dental practitioners have a very narrow and outdated conceptualisation of prevention. Based upon a contemporary understanding of behaviour change, a radical shift in many practitioners' philosophy is needed before they can effectively engage in preventive activity. Another key challenge is developing team working both within the dental setting but also with other colleagues across primary care. In England the new dental contract provides an opportunity to encourage greater levels of involvement in prevention. However appropriate training, the provision of relevant resources and on-going local support will all be needed to facilitate a reorientation to prevention. Undoubtedly PCDs will play a significant role in any future expansion in prevention within primary dental care settings.

R. G. Watt, Professor in Dental Public Health, University College London

DOI: $10.1038 / s j . b d j .4813771$ 